\title{
TRANSITION TO CHAOS IN THE DAMPED \& FORCED NON-LINEAR OSCILLATOR
}

\author{
Javier Montenegro Joo ${ }^{\mathrm{a}, \mathrm{b}^{*}}$ \\ ${ }^{a}$ VirtualDynamics Virtual Labs: Science \& Engineering, Calle Catorce, No 572, Las Magnolias del Surco, Lima 33, Perú. \\ ${ }^{b}$ Facultad de Ciencias Físicas, Universidad Nacional Mayor de San Marcos Ap. Postal 14-0149, Lima 14, Perú
}

(Recibido Marzo 31, 2009; Aceptado 18 Noviembre, 2009)

\begin{abstract}
A Virtual Lab to study the Transition to Chaos in second order non-linear differential equations has been developed and successfully applied to the search for chaotic behavior in the damped and forced non-linear oscillator. This simulation and visualization software evaluates the equation under investigation at up to one million time-steps, generating in real-time and on the screen, plots like amplitude of oscillation, phase diagram, amplitude oscillation peaks and an animation of an oscillator governed by the problem equation. In this way the investigator not only gets important behavior graphs but he or she also gets a physical visualization of the system under investigation. Visualizing an animation of the system under study is an enormous help because it is not always easy to interpret behavior graphs.
\end{abstract}

PACS: 05.45.-a; 05.45.Gg

Keywords: Virtual Lab, non-linear systems, oscillations, phase diagrams, chaos.

\section{Resumen}

Se ha creado un Laboratorio Virtual para estudiar la transición al caos en ecuaciones diferenciales no-lineales de segundo orden. Este software ha sido exitosamente aplicado a la investigación de la transición al caos en un oscilador no-lineal, amortiguado y forzado. El software, de simulación y visualización, evalúa la ecuación que se esta estudiando, en hasta un millón de puntos, generando en tiempo-real y en la pantalla del monitor, graficas de amplitud de oscilación, diagrama de fase y picos de amplitudes, produce además una animación del oscilador que se estudia. De esta forma, el investigador obtiene no solo importantes graficas de comportamiento del sistema que investiga, sino que también puede visualizarlo. La visualización de una animación del sistema que se estudia, es una enorme ayuda, pues no es siempre fácil interpretar graficas de comportamiento.

Palabras claves: Laboratorio virtual, sistema no-lineal, oscilaciones, diagrama de fase, caos.

\section{Introduction}

Oscillatory phenomena are everywhere in real life, the list of examples is never-ending, they can be found from heartbeats, and pulsating neurons, to earthquakes and pulsating stars. In some topics of social sciences and economy these phenomena are present too. There are also oscillating electrical circuits and oscillating chemical reactions and oscillations in atoms and molecules and in electromagnetic waves. Tides in the sea are also an example of oscillatory motion. Man-made engineering structures like bridges and machines may behave also as oscillators under some conditions.

\footnotetext{
* Correspondig author. e-mail: Director@VirtualDynamics.Org
}

Facing the above described scenario the investigation of oscillatory phenomena is very important.

The formal study of oscillations is based on models, being the simplest ones the swinging pendulum and the oscillating spring, both in vacuum (free oscillators). However, free oscillating pendulums and springs are rather ideal linear oscillators, this because real life oscillatory mechanisms are to some extent non-linear and subject to some kind of friction (damping). Besides this, usually there is also an external force acting upon these systems, so as to maintain its motion. Forced oscillating systems include at least two oscillating frequencies, one corresponding to the oscillator itself (its natural frequency) and another, 
associated to the external applied force. Hence there are -in the simplest case- two competing frequencies. It is well-known that after some initial time the oscillator winds up oscillating with the frequency of the external applied force. It is also known that dissipative systems with two competing frequencies exhibit transitions to chaos [1,2]

Resonance is common in oscillatory systems and when designing oscillatory mechanisms, engineers try by all means to avoid that so as to keep away from undesired harmful out-of-control vibrations which may eventually wreak havoc.

Under some conditions non-linear oscillators may display irregular and really complex oscillations, being it impossible to detect its period and amplitude of oscillation, in other words, the oscillation becomes aperiodical (it never exactly repeats).

Since the 1980's researchers have made enormous efforts to understand aperiodic motions of oscillatory systems, these endeavors have resulted in the Chaos Theory [2, 3]. Aperiodicity of non-linear dynamical systems is also known as Chaotic Dynamics. Chaos theory would have been fathered by Henri Poincare in the 1890 's, but he had no computers.

Concerning the organization of this paper, next an introduction to chaos is presented, then a description of the created software is exposed and after that the application to the case of the damped non-linear oscillator with variable damping is reported

\subsection{Common Chaos and Physical Chaos}

While everyday language understands chaos as complete disorder, tantamount to total randomness, it has a much subtler meaning in physics.

Chaos is the phenomena related to the occurrence of randomness and unpredictability in completely deterministic systems, it is not to be equated simply with disorder, it is more appropriate to consider Chaos as a kind of order without periodicity [4].

Stochasticity, Dynamical Chaos or simply Chaos $[5,6]$, is the appearance of apparently random motion in a deterministic dynamical system, this is, a system with no random forcing.

Even at long times chaotic systems include elements of order, this is, chaos is not completely random $[6,7]$.

A chaotic system is not a random system, it is deterministic. A chaotic system seems random if the beholder fails to recognize that it is chaotic. In a random system it is impossible to determine future states from previous states, in deterministic systems, that prediction is possible, provided the system is not sensitive to small variations in initial conditions.

\subsection{Period Doubling Route to Chaos.}

Several routes to chaos have been observed, however, the Period Doubling Route to Chaos (PDRC) $[8,9]$ is a universal and fundamental form of transition from periodicity to chaos (Transition to chaos [10]), observed in many mathematical and real systems.

In the Transition to Chaos [10], a system evolves toward non periodic time dependence as one or more parameters are varied.

In the PDRC [10] process, the time it takes the system to repeat itself, this is its Period, doubles and then doubles again and again, until the period becomes essentially infinitely long, as one or more control parameters (temperature, velocity, force, etc) are varied. Hence the PDRC involves an infinite sequence of Bifurcations [10], which appears as a Cascade of Bifurcations. All systems that become chaotic by the period doubling process do so in the same universal way. Beyond the period doubling cascade the stable periodic orbit disappears and chaos dominates the scene. However it is important to mention that not all systems evolving to chaos follow the bifurcation cascade behavior.

\subsection{Strange Attractor}

A Strange Attractor [10] is the shape (geometrical object) depicted in Phase Space (a.k.a. State Space) by a chaotic system. These attractors are fractal (have non-integer dimension) and consist of an infinitely number of closely spaced layers. Nearby trajectories diverge from each other by an amount that is exponential in time rather than proportional to time.

\subsection{Dependence on Initial Conditions}

A quality that characterizes chaotic systems is that these definitively show a sensitive dependence on initial conditions. Other systems may or may not exhibit this sensitivity, but chaotic ones definitively do. Sensitivity to initial conditions however, does not automatically entail chaos

\section{The Virtual Lab}

A Virtual Lab to numerically solve by the RungeKutta algorithm a second order non linear differential equation has been developed, this software can evaluate the equation in up to one million $\left(10^{6}\right)$ time steps, it shows the Time Series and the Phase Diagram (Phase Space) and detects and plots on screen the peaks and valleys of the amplitudes, all in a single screen and taking no more than 6 seconds in a Dual-Core Processor PC. Additionally a pendulum in motion according to the data generated in the simulation may be appreciated at the click of a button. This pendulum however, is just to visualize 
the oscillations; the problem under study may have nothing to do with a pendulum.

The two most important products of this software are the plotting on screen of the Bifurcation Cascade route to chaos of the equation it is solving and the animated pendulum, in this way, the route to chaos and chaos itself are easily and quickly visualized.

Seeing that dealing with non-linear systems is not straightforward at all, determining the parameters that generate the transition to chaos for a given differential equation is not an easy undertaking, and one way of detecting them may be by trial-and-error. If this program were set to operate on a trial-anderror mode search for chaos over randomly generated parameters, it would be able to investigate up to 20 to 30 different cases every minute (a huge time saving), and by virtue of a pattern recognition technique operating on phase space, automatically might report chaos when encountered.

\section{The Non-Linear Damped \&Forced Oscillator}

The equation under investigation is

$$
\frac{d^{2} x}{d t^{2}}+2 b \frac{d x}{d t}+\omega_{o}^{2} \operatorname{Sin} x=F_{o} \operatorname{Sin} \Omega t
$$

corresponding to a non-linear oscillator (a pendulum) immersed in a dissipative medium of damping constant $b$ and which is connected to an external applied sinusoidal force Fo oscillating with frequency $\Omega$, the free angular frequency of the linear oscillator is $\omega_{o}$.

It is expected that after a transient stage the oscillator will be forced to oscillate with the frequency $\Omega$ of the applied external force.

In view of the fact that the system under investigation is subject to dissipation (damping b) and since there are two competing frequencies, it is expected to observe a transition to chaos [1]. In the investigation reported in this document, the applied force Fo was slowly increased, which is equivalent to slowly lowering the damping.

\section{Results of the investigation}

Based on both experience and physical intuition the set of parameters where the oscillator under study shows a transition to chaos was successfully determined with the help of the developed software and after just a few trials, the result is shown in fig. 1, corresponding to 900,000 time steps. In Fig 1, region (a) corresponds to a period 1, region (b) corresponds to the first bifurcation, with period 2 , in region (c) a second bifurcation is displayed, with period 4 , and this is followed by region (d) with chaos. There must be another bifurcation after region (c) but it is not evident with the plotting resolution used.

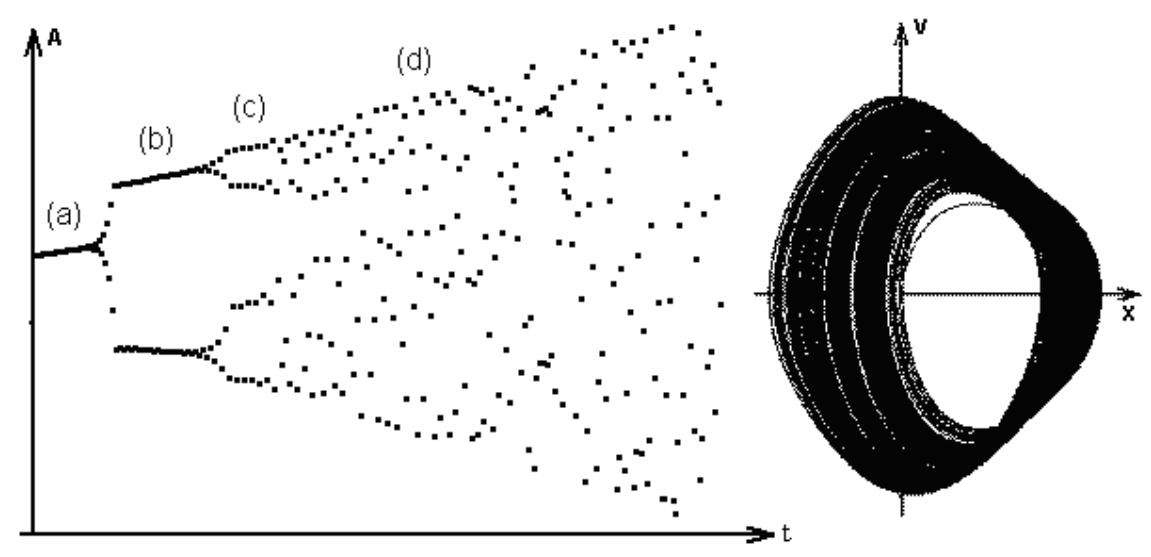

Fig. 1. Amplitude peaks vs. time: Two bifurcations are clearly seen, before chaotic motion sets in. The corresponding Phase Diagram (velocity vs. position) is also shown.

Fig. 2 shows the phase diagram for the first 530000 time steps of the simulation shown in Fig. 1, this is, from the start up to the point where the two first bifurcations in Fig. 1 are still easily distinguishable, right before the onset of chaos. It can be seen that the graph has self similarity; hence it may be a fractal.

Fig. 3 displays the oscillation amplitude peaks and valleys for a simulation along one million time steps, as the applied force Fo increases. The cascade of bifurcations is clearly seen. 
Fig 4 shows the Return Map for the case shown in Fig. 3. The Return Map is a plotting of $X_{n+1}$ as a function of $X_{n}$, where $X$ is the oscillation amplitude peak. It can be seen that for some values of $X_{n}$, there are several possible values of $\mathrm{X}_{\mathrm{n}+1}$, which means that there is impossible to predict an amplitude of oscillation.

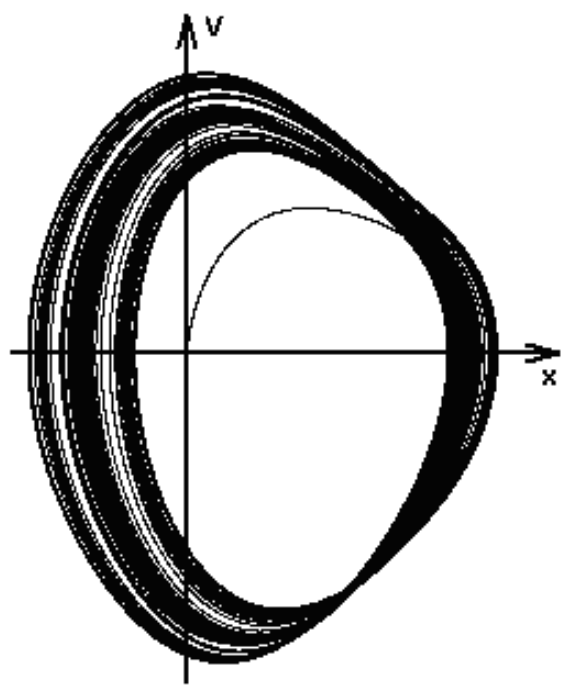

Fig. 2. Phase Diagram for the first $530000(\mathrm{x}, \mathrm{V})$ points of the simulation corresponding to Fig. 1, before the onset of chaos.

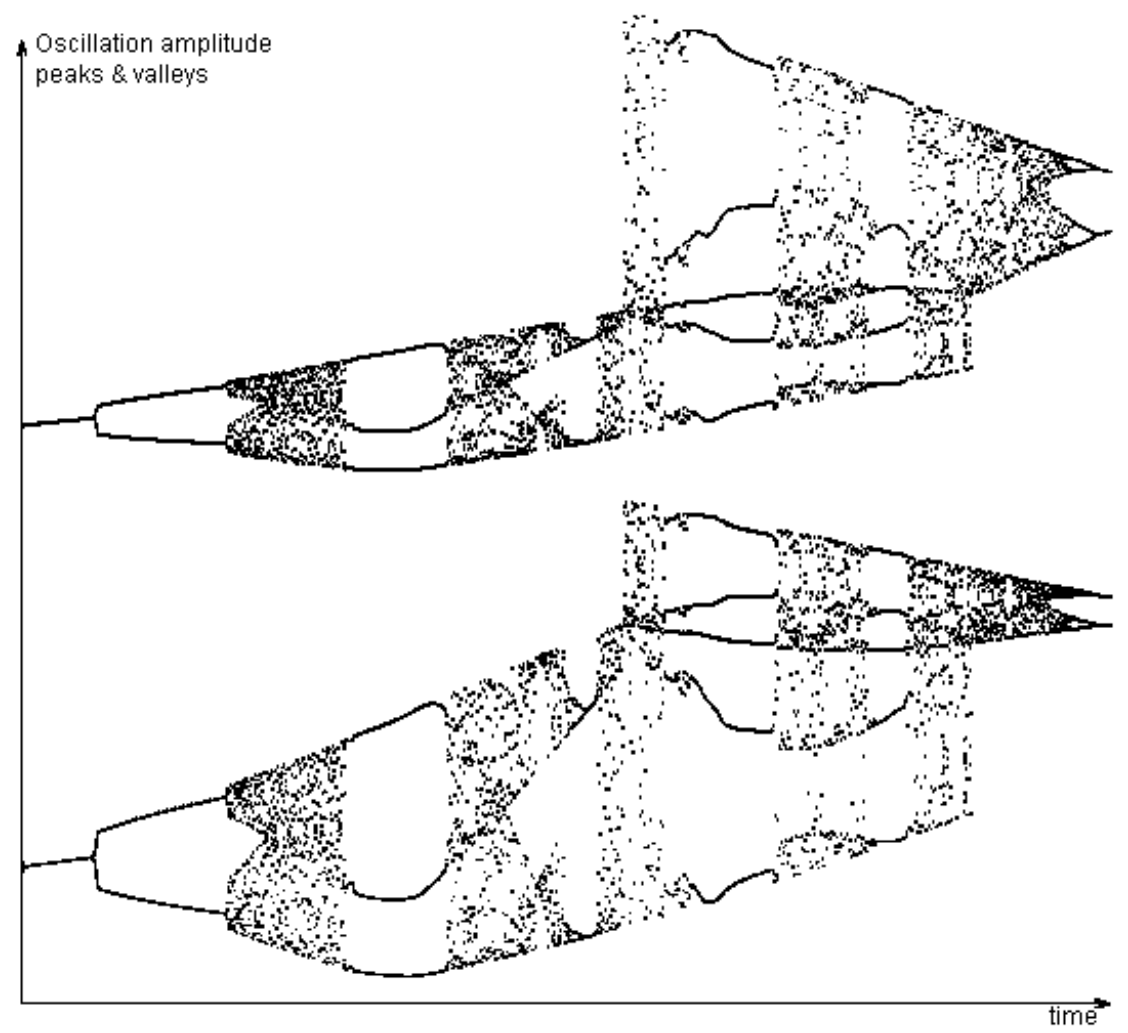

Fig. 3. Bifurcation cascade of the non-linear damped and forced oscillator. The graph shows the oscillation amplitude peaks (top) and valleys (bottom) versus time, for 1000000 time steps white applied force increases. 


\section{Conclusions}

The creation of a Virtual Lab to investigate the transition to chaos in second order non-linear differential equations has been reported. The Virtual Lab has been successfully applied to investigate the transition to chaos in the damped and forced nonlinear oscillator, with variable (increasing) applied force. The created software allows for a very quick simulation and visualization, reporting the Bifurcation Diagram, the Return Map, The Phase Diagram, and a visualization of the oscillations of the system under study

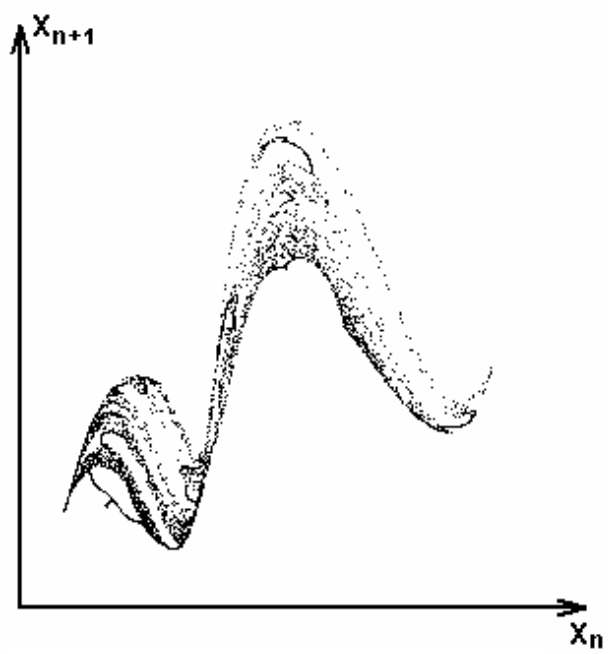

Fig. 4. Return Map for the case shown on Fig.3.

\section{References}

[1]. Mode-locking and the transition to chaos in dissipative systems. Per Bak, Tomas Bohr, Mogens H. Jensen, Physica Scripta, Vol T9, 5058,1985

[2]. Chaos and Nonlinear Dynamics, Robert C. Hilborn, Oxford University Press (pre print).

[3]. Synchronization and Control of Chaos, an Introduction for Scientists and Engineers, J.M. Gonzales-Miranda, Imperial College Press, 2004

[4]. Chaos, Hao Bai-Lin, World Scientific. Singapore, 1985

[5]. Chaos: How regular can it be?. ChernikovSagdeev-Zaslavsky, Physics Today, Nov. 1988.

[6]. The Mathematics of Chaos, Gary Taubes, Discover, Sept. 1984.

[7]. Chaotic Dynamics and the Origin of Statistical Laws, George M. Zaslavsky, Physics Today, August 1999.

[8]. Quantitative universality for a class of nonlinear transformations. J. Stat. Phys. Feigenbaum, M. (1978)

[9]. Universal behaviour in nonlinear systems, Physica D 7, Feigenbaum, M. (1983)

[10]. Chaos, A glossary. Schewe, Phillip. American Institute of Physics. 1985

\section{The Author}

Javier Montenegro Joo (JMJ) simultaneously studied both Physics and Computation (Computer Science) at San Marcos University (Lima, Peru), graduating as a Bachelor in Physics. He worked as scientific-systems analyst and computer programmer at the Peruvian Institute of Geophysics. JMJ obtained a Master of Science degree with a major in Computational \& Simulational Physics (The Ohio University, USA), and a Doutoral Qualificação in Cybernetic Vision (University of São Paulo, São Carlos, Brazil). JMJ is a Physics faculty member at San Marcos University, he is an Associate Member of the ICTP (Italy), and he is founding director of VirtualDynamics Virtual Labs: Science \& Engineering. 